\title{
Competition for big time opens
}

\author{
The launching of the Hubble Space Telescope next year will provide up to 3,000 hours of the best-ever \\ telescope time. Here is how to bid for some of it.
}

ThOsE hoping for a share of the time that will be available to users of the Hubble Space Telescope will at least now know to which address to write*. Towards the end of last month, the Space Science Institute on the Johns Hopkins campus at Baltimore put in the mail some thousands of copies of its formal Call for proposals, a document that explains the procedure applicants for telescope time must follow, together with more than a kilogram of supporting data. not to mention application blanks (twelve copies of which must be returned).

The documents give as full an account as there could be of how the potentially contentious issue of time allocation will be dealt with by the Space Science Institute. They are also, in passing, a vivid reminder of the potential power of the Hubble telescope, a 2.4-metre reflector equipped with instruments sensitive to radiation from the infrared $(11.000$ angstroms) to the ultraviolet (1.150) ångstroms).

That the instrument will do more for observational astronomy than the building of the Hale telescope on Palomar Mountain in the 1930)s seems generally accepted; it could yet outdo Galileo's contribution to the craft of observation. So competition for telescope time will be fierce.

So why will there be only $3,(0)(0)$ hours, rather less than 10 hours a day, of observing time each year? The new documents make plain the uncertanties that persist, the chief of which is that no date has yet been fixed for launching the telescope by means of the US space shuttle. And in the nature of things, nobody can guarantee that the instrument will find its way into orbit (at an altitude of $5(0)$ kilometres with an inclination of 28.5 degrees).

But the documents also explain that the efticiency with which the telescope can be reoriented towards novel targets is still an unknown quantity, whereas observations will not be feasible while the axis lies too close to the direction of the Sun, or the bright limbs of the outline of the Earth.

Applicants will also have to take account of the annoying complication of the South Atlantic Anomaly, the departure from regularity of the Earth's magnetic field that allows charged particles trapped in the magnetosphere to reach down

* General Observer Support Branch. Space Telescope Science Institute. PO Box 33139. Baltimore. Maryland 21218. USA. into the orbit of the telescope: the telescope detectors will be unusable not merely during passage through the anomaly but for an unquantifiable length of time thereafter, while the phosphorescence of the detectors makes the noise unacceptably high. One consequence is that the longest period of time when the continuous viewing of a target will be possible is estimated to be between 10 and 12 hours, a little more than at a high-latitude terrestrial observatory on a clear winter night.

The opportunities, on the other hand, are immense. The focal plane of the mirror is equipped with a variety of instruments, to each of which is assigned the radiation intersecting a predetermined patch of the plane, allowing the remainder of the radiation collected by the telescope to be gathered into the synoptic images of the wide-field camera (with the comparatively small field of view 154 arc-seconds square). This instrument will be able to acquire images, always in digital form, with a visual magnitude of 28 . Perhaps more striking is the expectation that the use for astrometry of one of the three fine guidance sensors with which the telescope is equipped (two are needed for pointing the telescope) will allow angular positions to be determined to within $0.0(0) 16$ arc seconds. This expectation, if borne out by experience, implies that it will be possible directly to determine the distances to important milestone stars used in constructing the distance scale of the Universe.

So how are these great opportunities to be shared? In principle, according to the Space Science Institute, anybody may apply for time. All proposals will be put through the same peer-review system, and time allocated on merit.

On the teasing question of deciding between the merits of proposals yielding quick spectacular results and long-term programmes bearing fruit only after the accumulation of a mass of data, the institute plans an arbitrary but workable solution. Each proposal wil be considered by a disciplinary review panel, but final recommendations will be made by a Telescope Allocation Committec with general instructions to allocate roughly equal amounts of time to proposals in the categories small, medium and large. These allocations will account for roughly 90 per cent of the time, with the remainder reserved for allocation by the director. among other things so that unexpected opportunities may be seized and so that the telescope may be used in novel ways.

The institute has already settled on three key projects to which special attention will be given, of which the chief is the determination of the distance scale, ideally so as to determine Hubble's constant to within ten per cent. The second consists of an invitation to exploit the potential of the wide-field camera so as to carry out a deep survey of selected regions of the sky, yielding information on such miscellaneous issues as the comet cloud beyond Neptune and the supernova rate in distant galaxies. Quasar absorption lines come third.

Speed will obviously be crucial. The documents make plain that applications for time will not succeed if the objective is a project identical with one already in the programme. Thus the first convincing proposal to measure Hubble's constant is likely to hold sway for two or three years. The other side of this coin of exclusivity is the institute's policy on the publication of data. At the outset, an observer will have exclusive access to the data gathered on his behalf for twelve months after the last of them have been stored, but thereafter. the data will be become part of the general archive at Baltimore.

So how should intending applicants proceed? First, read the instructions carefully. The chances of being caught out by misunderstanding the technical performance of the instruments, or by miscalculating the length of the exposure required, are high. Those who succeed in the first round of applications will find themselves quickly asked for accurate positions of the guide stars they specify for their targets. Perhaps the best hope of winning time early is to go carefully through the list of projects put forward by those guaranteed exclusive use of the first six months (people who have helped to develop the telescope), looking for opportunities to make parallel observations of objects within the field of view of the Hubble telescope during that initial period.

But can it be worth the trouble when there is always a risk that the telescope may not be launched successfully? The risk of failure is no greater than that the shuttle itself might fail. But, whatever happens, the list of proposals on their way to Baltimore in the next three months is likely to serve future historians well as a record of the ambitions of the world's astronomers at this interesting time.

John Maddox 